\title{
Does Over-the-Counter Purchase of Antihistamines by Residents of Dhaka City, Bangladesh Align with the Prescribing Choices of the Physicians Practicing in That City?
}

\author{
Somaia H. Chadni, ${ }^{1}$ Md. H. A. Banna, ${ }^{2}$ Nafisa N. Mollick, ${ }^{2}$ Md. R. I. Rupam, \\ Sharmin Sultana, ${ }^{3}$ and Shadid U. Zaman ${ }^{2}{ }^{2}$ \\ ${ }^{1}$ Department of Pharmacy, Comilla University, Comilla 3500, Bangladesh \\ ${ }^{2}$ Department of Pharmacy, Northern University Bangladesh, Dhaka 1205, Bangladesh \\ ${ }^{3}$ Department of Pharmacy, University of Asia Pacific, Dhaka 1205, Bangladesh \\ Correspondence should be addressed to Shadid U. Zaman; zaman.shadid@nub.ac.bd
}

Received 4 October 2019; Revised 19 November 2019; Accepted 26 November 2019; Published 21 January 2020

Academic Editor: Jagdish Khubchandani

Copyright (c) 2020 Somaia H. Chadni et al. This is an open access article distributed under the Creative Commons Attribution License, which permits unrestricted use, distribution, and reproduction in any medium, provided the original work is properly cited.

\begin{abstract}
Most current guidelines recommend prescribing second-generation antihistamines (SGAs) over first-generation antihistamines because SGAs are less likely to cause sedation and impairment of heavy work performance. However, common residents who use these antihistamines as over-the-counter (OTC) medicines are less likely to know that. So, this study was designed to compare the over-the-counter use of antihistamines by common residents with the prescribing preferences of physicians residing at Dhaka City, Bangladesh. Between June and August of 2017, a total of 100 Physicians from some of the top medical institutions of the city and 350 randomly selected common residents were directly interviewed with two separate semistructured questionnaires specifically designed for each population. Data was statistically analyzed using Fischer's exact test, Spearman's rank correlation test and Kendall's tau rank correlation test. The data shows that physicians prefer second-generation antihistamines with fexofenadine (48.09\% of the total responses), desloratadine (16.03\%), and rupatadine (13.74\%) taking the top spots. Cetirizine (29.46\% of total responses), desloratadine (14.73\%), and chlorpheniramine (14.52\%) were the most used OTC antihistamines by the common residents. Statistical analysis with Fischer's exact test revealed that the difference in preference of first-generation antihistamines between physicians and common residents were extremely significant $(p<0.0001)$. Furthermore, cetirizine (which is known to have some degree of sedating activity) and chlorpheniramine are more preferred among common residents than among physicians (extremely significant difference, $p<0.0001$ in both cases). The study concludes that physicians of Dhaka City are complying with practice guidelines, but sedating antihistamines still retain some popularity among the common residents. Hence, a more engaging community pharmacy is needed to minimize adverse effects that can arise from OTC use of sedating antihistamines.
\end{abstract}

\section{Introduction}

Bangladesh is a developing country with a very high population density. As a consequence, it has been difficult to provide the best healthcare to the mass. However, the pharmaceutical sector of the country is quite advanced and has been able to fulfill the country's need of medicines [1]. This has created another problem, as the wide availability of medicines has prompted the common people and sometimes the physicians to unnecessarily resort to certain medicines $[2,3]$. This is further complicated by the lack of awareness about the dangers that accompanies unsupervised use of medicines. Recently, regulations have been developed to battle the indiscriminate use of medicines, but they are yet to be enforced effectively [4].

Antihistamines are a class of drugs which are commonly taken by the common residents as over-the-counter (OTC) medicine. These drugs are also frequently prescribed by physicians for colds and various types of allergic conditions. Antihistamines are generally considered to be safe, and are recognized as OTC medicine in most parts of the world. However, among the two classes (first-generation and 
second-generation) of antihistamines available in the market, the first-generation antihistamines (FGAs) can possess some risks to the patients due to their sedative property.

Multiple research organizations and medical practitioners' networks now recommend the use of second-generation antihistamines (SGAs) as the first choice in place of FGAs. In 2009, a joint initiative consisting of the European Academy of Allergology and Clinical Immunology (EAACI), Global Allergy and Asthma European Network (GA ${ }^{2}$ LEN), European Dermatology Forum (EDF), and World Allergy Organization (WAO) made a recommendation that FGAs should not be used for urticaria and other allergic disorders and that recommendation was kept in the revised guideline published by the joint venture $[5,6]$. In 2010, GA ${ }^{2}$ LEN published a position paper which actually recommended that FGAs should no longer be available as OTC products due to their various side-effects and availability of better alternatives (SGAs) [7]. The American guideline developed by Joint Task Force on Practice Parameters (JTFPP) consisting of the American Academy of Allergy, Asthma and Immunology (AAAAI) and the American College of Allergy, Asthma and Immunology (ACAAI) has similar recommendations giving emphasis on SGAs over FGAs [8]. The World Health Organization (WHO) and Allergic Rhinitis and its Impact on Asthma (ARIA) guidelines also recommends SGAs [9]. In all instances, the main concern has been the sedative effect of FGAs and its consequences. Studies have shown that FGAs can disrupt REM (Rapid Eye Movement) sleep and impair cognitive function [10]. Furthermore, it also impairs the user's ability to perform heavy work and the ability to drive [11].

This work describes a survey-based study initiated to identify which antihistamines are being commonly taken by residents as OTC medicines and which are commonly prescribed by the physicians at Dhaka City of Bangladesh. Such information can be used to understand if the patients are taking antihistamines which possess greater risks to them. It would also allow the evaluation of the physician's role in the usage pattern of antihistamines by common residents who purchase it as OTC medicine.

\section{Materials and Methods}

2.1. Survey Questionnaire Preparation. Survey questionnaire had to be prepared to collect data from two distinct populations namely the common people living in Dhaka City and the physicians who practice at the city. Consequently, two different survey questionnaire forms were prepared specifically to serve the purpose. The questionnaires were semistructured in nature so that a more robust statistical analysis could be performed but at the same time the respondents would be free to express their own opinions regarding the question asked. The questionnaire directed towards the common people was designed to learn about the actions taken by respondent when he/she contracted diseases like allergies and colds, the choice of antihistamine for such diseases, and the side-effects observed by the respondent. The questionnaire directed towards the physicians contained questions such as which class of antihistamines are more commonly prescribed by them, which side-effect is most concerning to them, and whether they support self-medication of antihistamines.

2.2. Data Collection. Data was collected from various locations within Dhaka City, Bangladesh between June and August of 2017. Physician's data were collected by direct interview from doctors working at top medical institutions of the City including Dhaka Medical College and Hospital, Shaheed Suhrawardy Medical College and Hospital and Sir Salimullah Medical College and Hospital. Physicians in the field of General Medicine, Obstetrics and Gynecology, and Surgery were purposively selected as they are expected to prescribe them. For the collection of data from common residents, respondents were directly interviewed by the surveyor who was trained to interpret the brand names because common residents are less likely to recognize generic names.

2.3. Ethical Review. This study was conducted in accordance with the Declaration of Helsinki (2000) for research involving human subjects. The two research proposals to survey physicians and the common residents were approved by the Research center, Department of Pharmacy, Northern University of Bangladesh under the condition of obtaining oral consent from the respondents and maintaining appropriate confidentiality of those individuals (Ref. No. DoP/RC/ EC/2017/05/app/01 and DoP/RC/EC/2017/05/app/02). Oral consent was obtained from all individuals prior to data collection.

2.4. Statistical Analysis. Collected data was tabulated by simple spreadsheet analysis. The data obtained from physicians and common residents was compared and the differences were evaluated using nonparametric tests namely the Fischer's exact test. The Spearman's rank correlation coefficient and Kendall's tau rank correlation test were used as a nonparametric measure of rank correlation between the choice of antihistamine among physicians and common residents. All analysis was performed in GraphPad Prism (version 7.00) except determination of Kendall's tau which was done using Minitab (version 8.0).

\section{Results}

3.1. Survey of Physicians. Physician survey was conducted on a total of 100 reputed physicians practicing at Dhaka City. Among them 59\% were General Medicine specialists, $13 \%$ were Surgeons, and 22\% were Obstetricians and Gynecologists. $90 \%$ of the participating physicians had more than 2 years of experience. It was observed that $95 \%$ of the surveyed physicians commonly prescribe antihistamines. Data shows that $76 \%$ of the surveyed physicians prescribe nonsedating antihistamines for most occasions whereas only $4 \%$ mostly prescribe first-generation antihistamines. Among the antihistamine molecules available in Bangladeshi Market, according to the survey the top choice of the physicians was fexofenadine (used by $66.32 \%$ of the total number respondents) followed by desloratadine $(22.11 \%$ of the total 


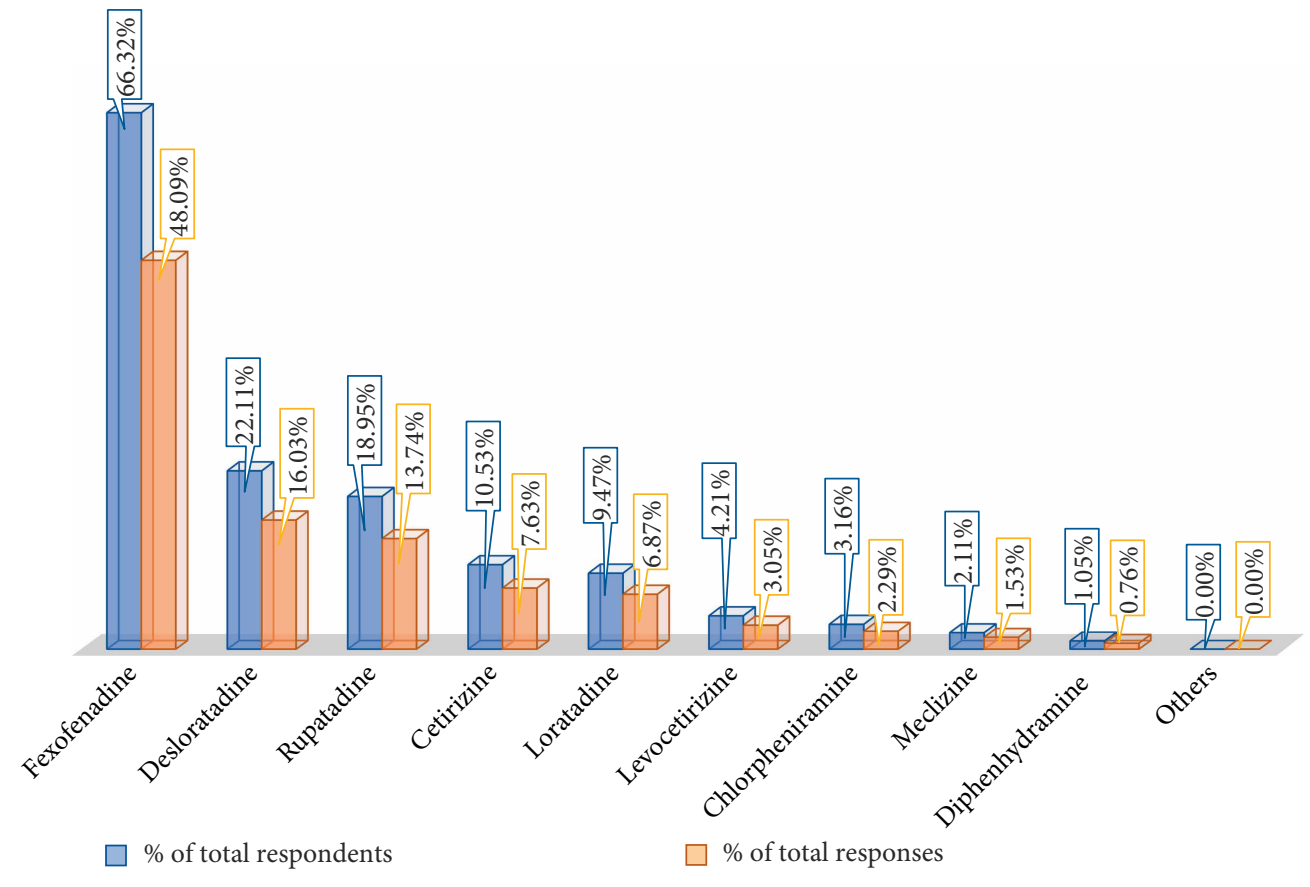

FIGURE 1: Prescribing choices of antihistamine molecules according to the survey on physicians. Multiple answers were accepted for this question. Hence, the percentage has been calculated on the basis of total number of responses as well as the total number of respondents.

number respondents), and rupatadine (18.95\% of the total number respondents). Figure 1 shows the data in terms of both the total number of respondents and the total number of responses.

The survey also revealed that physicians consider sedation to be one of the most concerning side-effects of antihistamines (Figure 2).

3.2. Survey of Common Residents. The survey of common residents was conducted on 350 randomly selected individuals residing at Dhaka City. Most of these individuals were literate and came from middle-income families. One of the goals of the survey on common residents was to evaluate the extent of self-medication and the data shows that $71.14 \%$ of the respondents resorted to self-medication and only $22 \%$ went to physicians for consultation for cold or allergies.

In this survey, the top antihistamine of choice was cetirizine, followed by desloratadine, and chlorpheniramine. Some other nonsedating antihistamines such as fexofenadine, and rupatadine were also mentioned (Figure 3 ). It appears that personal preference $(41.37 \%$ among the respondents who went for self-medication) is one of the most important factor when selecting a specific antihistamine for self-medication. But some (6.43\% among those who self-medicated) sought help from family members who are healthcare professional when selecting a specific antihistamine. Only a fraction (17.27\% of those who self-medicated) are using previous prescriptions to select the antihistamine for self-medication (Figure 4).

In case of side-effects, three-quarters (72.7\%) of the respondents who answered the question reported sedation (Figure 5). But it should also be mentioned that almost $40 \%$ did not report any side-effects (i.e. did not answer the question).

3.3. Statistical Comparison. When comparing the survey data from physicians and common residents, the choice of antihistamines in the two groups was one of the most interesting aspects. Nonparametric tests were used to determine if their choices are correlated or not. When performing these tests, only the choices that garnered a minimum of $5 \%$ response from either population were considered. The Spearman's rank correlation coefficient $(r)$ was found to be 0.086 indicating there is no correlation at all but the validity of this inference was statistically limited $(p=0.919)$. Similar observation was made with Kendall's tau rank correlation test $\left(\tau_{b}=0.067, p=1\right)$.

To study this dissimilarity between the two survey populations, the responses from the participants were grouped into two categories namely "sedating antihistamines" and "nonsedating antihistamines". Then, a two-sided Fischer's exact test was performed to determine whether any significant difference existed between the physicians and general public in terms of selecting sedating or nonsedating class of antihistamine. At $95 \%$ confidence interval, the $p$-value was less than 0.0001 indicating extremely significant result. This indicates that there is statistically significant difference in the choice of antihistamine class between physicians and the general public.

A test was also performed to see whether there is any significant difference in the popularity of chlorpheniramine between physicians and common residents and it was found that the difference was extremely significant ( $p$-value $<0.0001$ ). Similar result was obtained when the test was repeated with cetirizine ( $p$-value $<0.0001)$. 


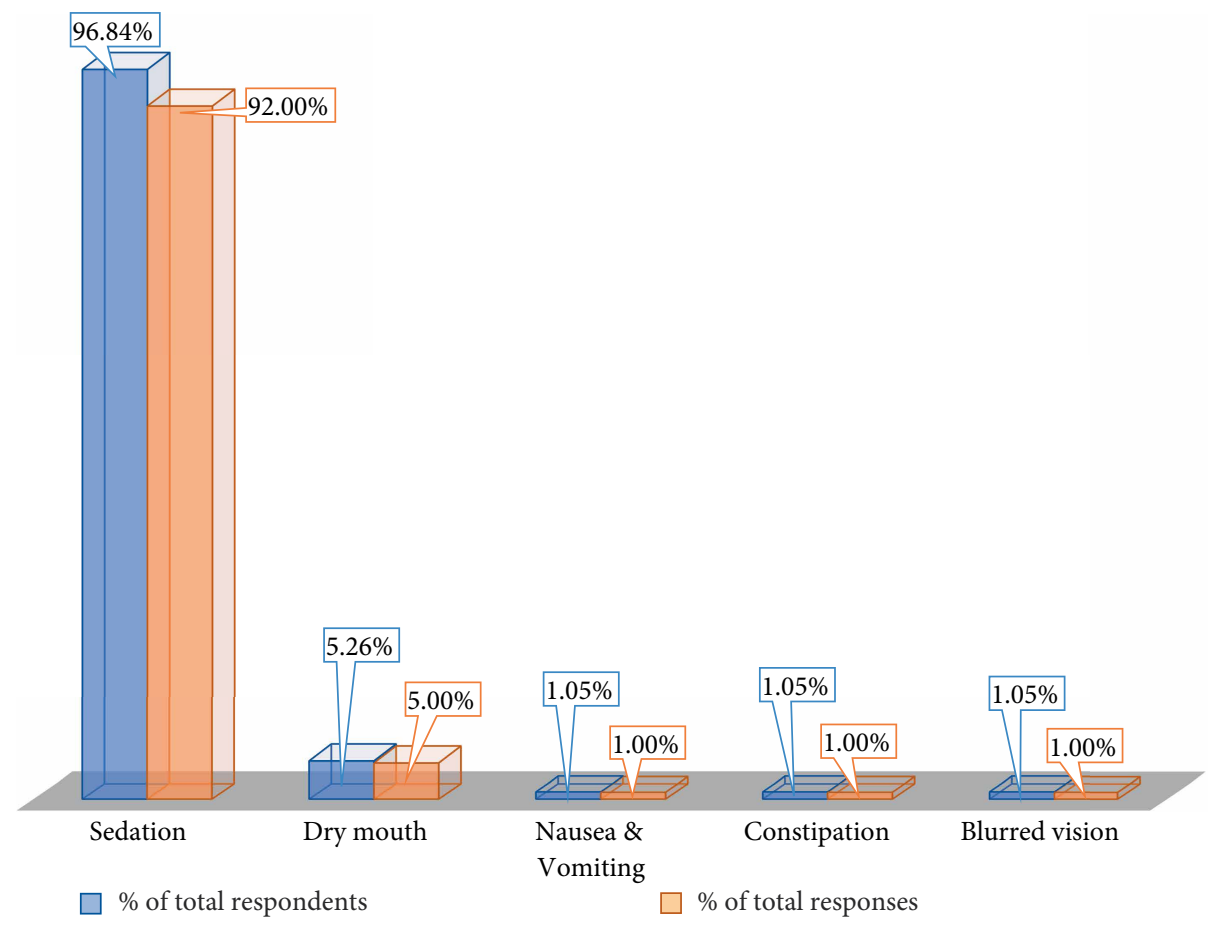

Figure 2: Most concerning side-effects of antihistamines in the opinions of surveyed physicians. Physicians were asked to mention the sideeffect which of the greatest concern to them, multiple responses were allowed, hence the percentage is calculated based on the total number of responses as well as the total number of respondents.

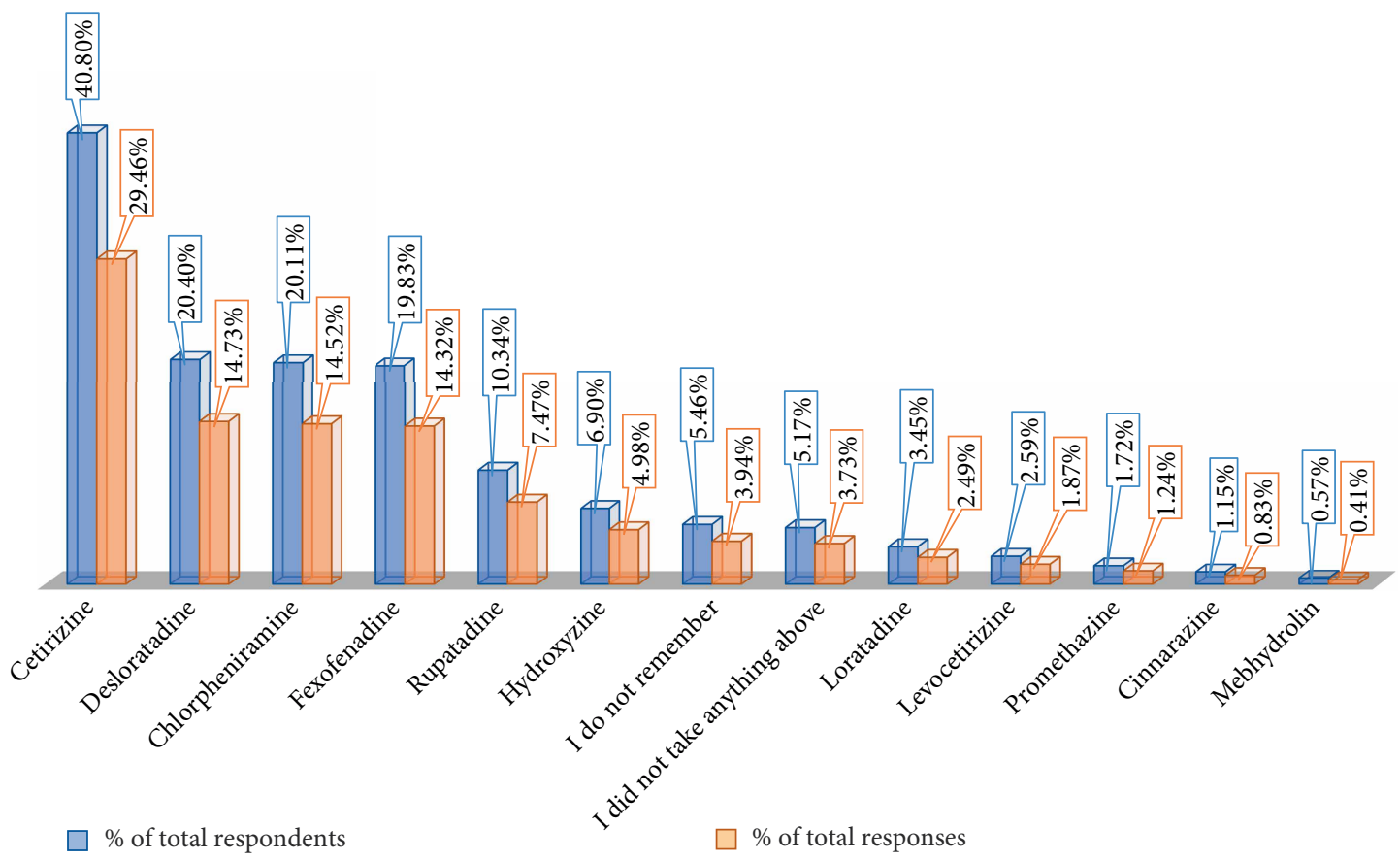

Figure 3: Choice of Antihistamine for OTC purchase by the common residents of Dhaka City. The Respondents were asked to name the antihistamines they normally purchase when a prescription is not available. Multiple responses were allowed for the question and therefore, the percentage is calculated based on the total number of responses as well as the total number of respondents. 


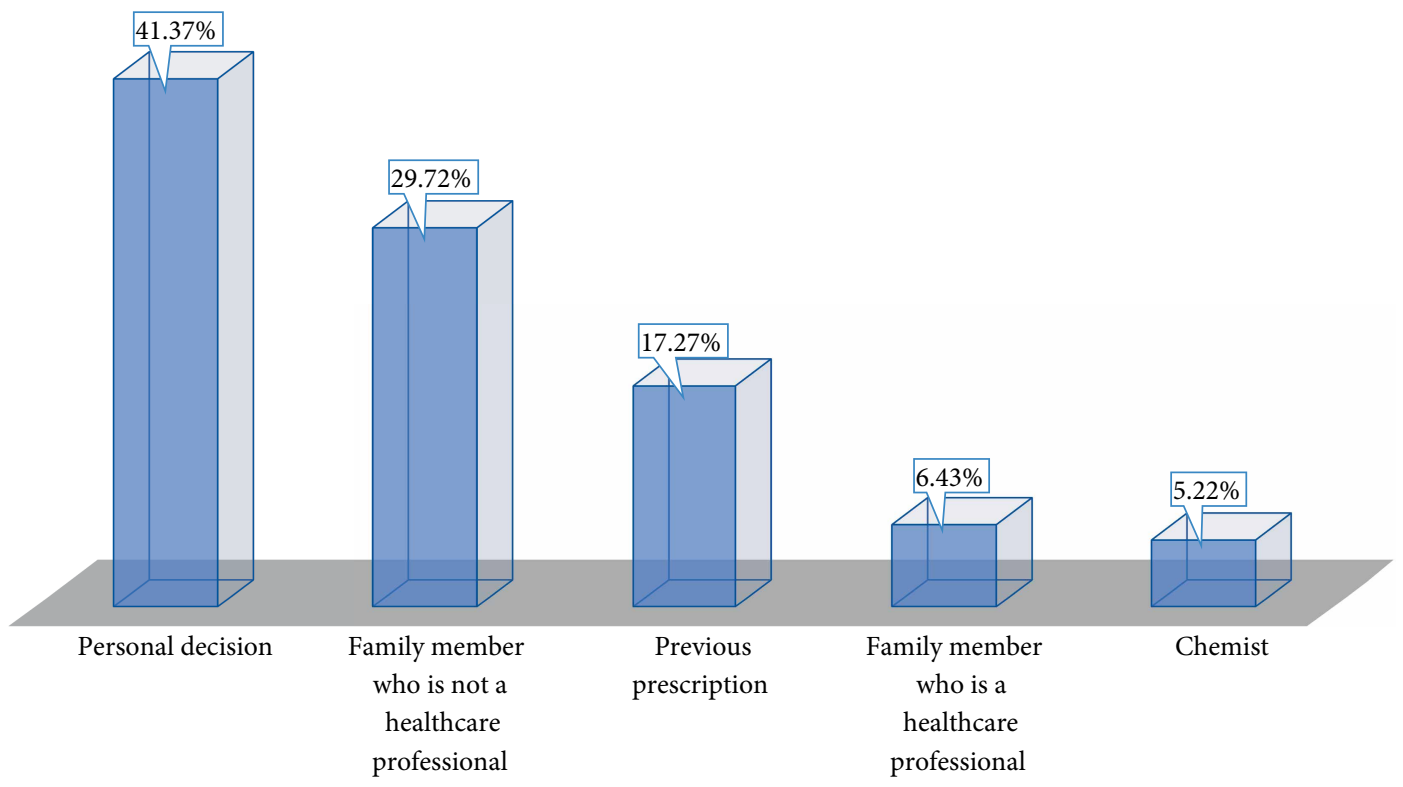

FIGURE 4: Sources of influence during selection of an antihistamine molecule for self-medication. For this question, only a single response was accepted. The chart shows the percentages of the total respondents.

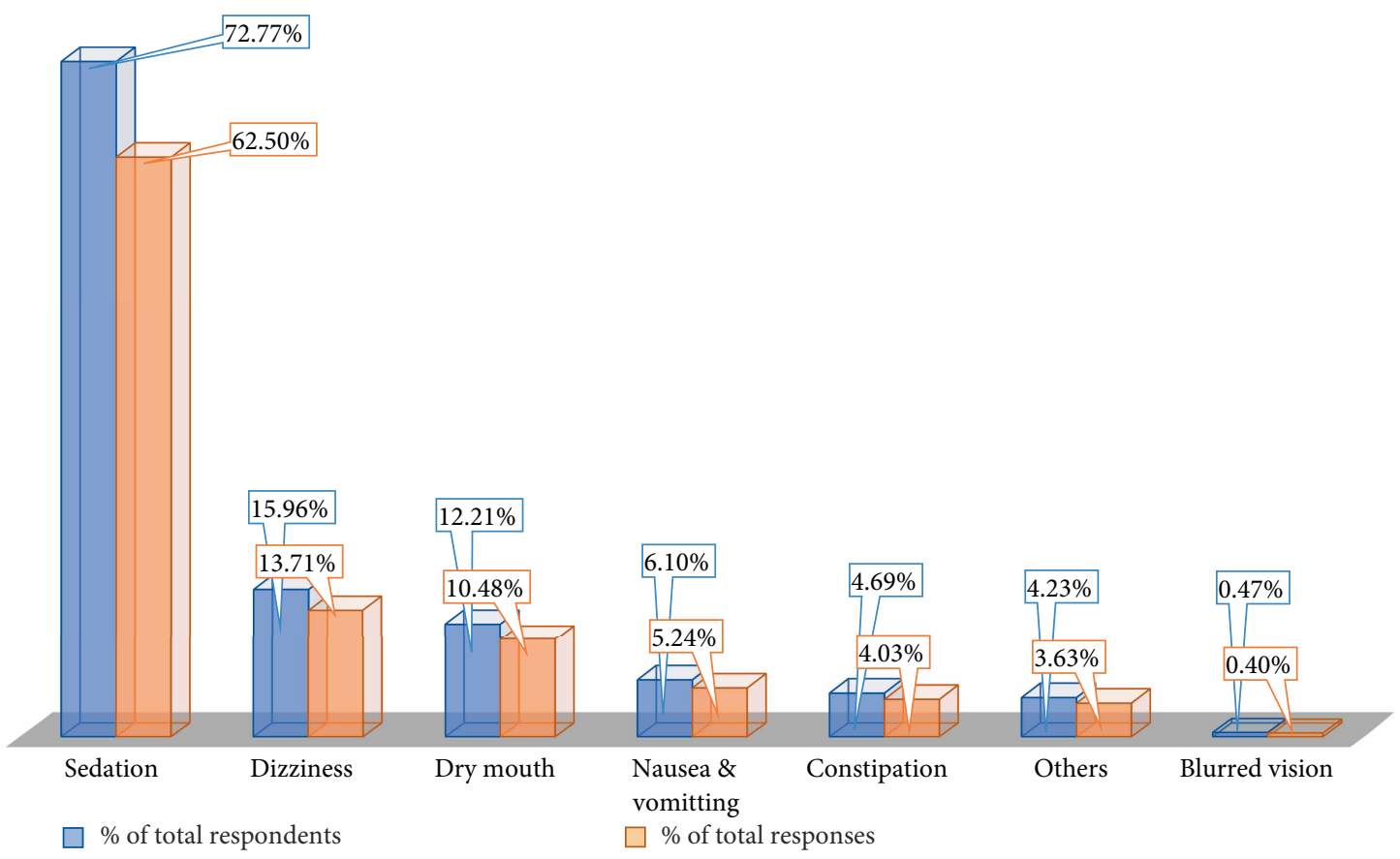

FIGURE 5: Most concerning side-effects of antihistamines according to the common residents. Respondents could provide multiple responses to this question.

\section{Discussion}

Antihistamines are commonly used by patients to revceive relief from allergic symptoms. In the USA, only first-generation antihistamines were available as over-the-counter product till 2001. A citizen petition motivated the Food and Drug Administration to make several SGAs available as OTC products as well [12]. Regardless of the original intentions behind the petition, various economy models showed that this actually reduced the overall cost of therapy by reducing the incidences of side-effects [12].

The main side-effect of concern for healthcare professionals has been the sedative property of this class of drugs. Sedation may be beneficial for some patients, but it renders 
TABle 1: Price of common first and second-generation antihistamines in Bangladesh (as of October, 2018).

\begin{tabular}{lccc}
\hline Antihistamine & Dose $(\mathrm{mg})$ & Price in BDT & $\begin{array}{c}\text { Price in USD } \\
\text { (approx.) }\end{array}$ \\
\hline Chlorpheniramine & 4 & 0.30 & 0.00375 \\
Hydroxyzine & 10 & 1.25 & 0.0156 \\
Promethazine & 10 & 0.68 & 0.0085 \\
Cinnarizine & 10 & 1 & 0.0125 \\
Mebhydrolin & 50 & $1.50-3.00$ & $0.01875-$ \\
Fexofenadine & 60 & 5.00 & 0.0375 \\
Desloratadine & 5 & 2.50 & 0.0625 \\
Rupatadine & 10 & 10.00 & 0.03125 \\
Cetirizine & 10 & $1.50-3.00$ & 0.0125 \\
\end{tabular}

BDT: Bangladeshi Taka, USD: United States Dollar.

them unable to perform work that requires psychomotor competency such as driving or lifting heavy objects. A study evaluated the impact of FGAs in aviation accidents and discovered that FGAs were found in $11 \%$ of the accidents in 2004 [7]. Various studies have also shown that FGAs tend to significantly impair driving performance, and perhaps some second-generation agents (e.g. Cetirizine and Loratadine) cause some degree of impairment as well $[10,11,13-15]$. One study found that patients using FGAs have 1.61 times the risk of traffic accident compared to nonusers but the significance of the finding was statistically limited [16].

Consequently, reduced use of FGAs by both physicians and common residents may hold some benefits in healthcare and society. This study has found that physicians practicing at Dhaka City have largely moved away from FGAs with only around $5 \%$ of the physicians still prescribing those. However, a significant percentage of the common residents is still using FGAs (30.29\% of the total respondents, $21.98 \%$ when considering the total number of responses rather than the respondents). A study conducted in Germany back in 2011 showed that while nearly half of the participating physicians use SGAs, $23 \%$ physicians were still prescribing FGAs [17]. So, the results obtained here is quite impressive.

It is also encouraging to see that physicians consider sedation to be one of the most concerning side-effects of antihistamines. This may have contributed to their preference of SGAs. A large fraction (44.29\% in terms of total respondents) of the surveyed common residents also experienced sedation as the main side-effect. Regardless, the reason why FGAs specifically chlorpheniramine are still being used may be that the price of these drugs are lower compared to available SGAs. The prices of some FGAs and SGAs are listed in Table 1. Since chlorpheniramine is 5 times cheaper than the cheapest SGA available in the country, it makes sense that it has some degree of popularity among the common residents. For anyone interested, they can visit the official website of Directorate General of Drug Administration (DGDA) of Bangladesh, to look up the current prices [18].

Another important finding was that common residents are using cetirizine significantly more when compared with the physicians prescribing it. Cetirizine is a second-generation antihistamine which has been a popular choice during the years 2000s. Cetirizine has been found to impair performance to some degree in some studies, although at a lower extent compared to FGAs $[10,15,19,20]$. Nevertheless, it has higher sedation potential compared to other second-generation antihistamines. So, it is interesting to see that only $10.53 \%$ of the surveyed physicians (7.63\% in terms of total responses) are prescribing cetirizine, but $40.80 \%$ (29.26\% in terms of total responses) of the common residents prefer it.

A potential factor that could had explained common residents' choice of purchasing sedating antihistamines like FGAs and cetirizine was their use as sleep aids. However, no antihistamine is approved in Bangladesh for use as a sleep aid. In fact, as the data in Figure 5 shows, $72.77 \%$ of the respondents consider sedation to be a concerning side-effect, which in the opinion of the authors, is indicative that the respondents do not view them as sleep aids. Furthermore, advertising or promoting medicines in a public platform is strictly prohibited in the country. Hence, the choice of antihistamines could not be significantly affected by those factors.

This study is not without some limitations, most importantly the small sample size, especially when surveying the common residents. Another thing to note is that multiple answers were allowed for various questions asked in the survey. This made statistical analysis of the data more complicated. However, it is the belief of the authors that allowing multiple answers was necessary to reflect real-life situation specially for the physician survey.

The study shows that the physicians practicing at Dhaka City have moved away from first-generation antihistamines and prefer to prescribe antihistamines which have nearly zero potential to cause sedation and other effects on the central nervous system. On the other hand, common residents who are using antihistamines over-the-counter are still continuing to use molecules which have some degree of sedative activity. Since the common residents are usually not using to the advice of healthcare professionals to select an antihistamine for over-the-counter purchase, it may take some time before the market makes a complete move to nonsedating antihistamines.

\section{Conclusion}

Sedating antihistamines i.e. first-generation antihistamines are no longer recommended by healthcare networks and guidelines due to the risks associated with impaired performance in physical activities like driving and heavy work caused by these drugs. At Dhaka City, which is the center of healthcare profession in Bangladesh, physicians are showing very good compliance with those guidelines. However, a fraction of the common residents still continues to use those sedating antihistamines over-the-counter. Therefore, the city requires more stringent practice of community pharmacy where skilled pharmacists would be available to provide the common residents with necessary advice regarding the use of antihistamines. 


\section{Data Availability}

The portion of the collected data that support the findings of this study are available from the corresponding author upon request.

\section{Conflicts of Interest}

The authors declare that they have no conflicts of interest regarding the publication of this paper.

\section{Acknowledgments}

This research was partially funded by the Research fund of the Department of Pharmacy, Northern University Bangladesh.

\section{References}

[1] J. Sultana, "Future prospects and barriers of pharmaceutical industries in Bangladesh," Bangladesh Pharmaceutical Journal, vol. 19, no. 1, pp. 53-57, 2016.

[2] M. S. Islam, "Therapeutic drug use in Bangladesh: policy versus practice," Indian Journal of Medical Ethics, vol. 5, no. 1, pp. 24-25, 2008.

[3] M. Mohiuddin, S. F. Rashid, M. I. Sbuvro, N. Nahar, and S. M. Ahmed, "Qualitative insights into promotion of pharmaceutical products in Bangladesh: how ethical are the practices?" BMC Medical Ethics, vol. 16, Article ID 80, 2015.

[4] Ministry of Health and Family Welfare, "National Drug Policy 2016," 2017, http://www.dgda.gov.bd/index.php/laws-andpolicies/204-national-drug-policy-2016-including-essential-druglist-and-otc-list/file.

[5] T. Zuberbier, W. Aberer, R. Asero et al., "The EAACI/GA²LEN/ $\mathrm{EDF} / \mathrm{WAO}$ guideline for the definition, classification, diagnosis and management of urticaria," Allergy, vol. 73, no. 7, pp. 1393$1414,2018$.

[6] T. Zuberbier, R. Asero, C. Bindslev-Jensen et al., "EAACI/ GA2LEN/EDF/WAO guideline: definition, classification and diagnosis of urticaria," Allergy, vol. 64, no. 10, pp. 1417-1426, 2009.

[7] M. K. Church, M. Maurer, F. E. R. Simons et al., "Risk of firstgeneration H1-antihistamines: a GA2LEN position paper," Allergy, vol. 65, no. 4, pp. 459-466, 2010.

[8] J. A. Bernstein, D. M. Lang, D. A. Khan et al., "The diagnosis and management of acute and chronic urticaria," The Journal of Allergy and Clinical Immunology, vol. 133, no. 5, pp. 1270-1277, 2014.

[9] J. L. Brożek, J. Bousquet, C. E. Baena-Cagnani et al., "Allergic rhinitis and its impact on asthma (ARIA) guidelines: 2010 revision," Journal of Allergy and Clinical Immunology, vol. 126, no. 3, pp. 466-476, 2010.

[10] E. E. Philpot, "Safety of second generation antihistamines," Allergy and Asthma Proceedings, vol. 21, no. 1, pp. 15-20, 2000.

[11] J. C. Verster and E. R. Volkerts, "Antihistamines and driving ability: evidence from on-the-road driving studies during normal traffic," Annals of Allergy, Asthma and Immunology, vol. 92, no. 3, pp. 294-304, 2004.
[12] P. W. Sullivan, K. V. Nair, and B. V. Patel, "The effect of the $\mathrm{Rx}$-to-OTC switch of loratadine and changes in prescription drug benefits on utilization and cost of therapy," The American Journal of Managed Care, vol. 11, no. 6, pp. 374-382, 2005.

[13] Y. Hu, D. E. Sieck, and W. H. Hsu, "Why are second-generation H1-antihistamines minimally sedating?" European Journal of Pharmacology, vol. 765, pp. 100-106, 2015.

[14] P. G. Ozdemir, A. S. Karadag, Y. Selvi et al., "Assessment of the effects of antihistamine drugs on mood, sleep quality, sleepiness, and dream anxiety," International Journal of Psychiatry in Clinical Practice, vol. 18, no. 3, pp. 161-168, 2014.

[15] S. R. Mohler, A. Nicholson, P. Harvey, Y. Miura, and S. G. Meeves, "The use of antihistamines in safety-critical jobs: a meeting report," Current Medical Research and Opinion, vol. 18, no. 6, pp. 332-337, 2002.

[16] A. Perttula, J. Pitkäniemi, O. P. Heinonen et al., "Secondgeneration antihistamines exhibit a protective effect on drivers in traffic-a preliminary population-based case-control study," Traffic Injury Prevention, vol. 15, no. 6, pp. 551-555, 2014.

[17] K. Weller, K. Viehmann, M. Bräutigam et al., "Management of chronic spontaneous urticaria in real life-in accordance with the guidelines? across-sectional physician-based survey study," Journal of the European Academy of Dermatology and Venereology, vol. 27, no. 1, pp. 43-50, 2013.

[18] Directorate General of Drug Administration Bangladesh, "Directorate General of Drug Administration: allopathic drug database. Search price," 2018, http://www.dgda.gov.bd/ indexp.p/search-price.

[19] M. Ferrer, M. Morais-Almeida, M. Guizova, and R. Khanferyan, "Evaluation of treatment satisfaction in children with allergic disease treated with an antihistamine," Clinical Drug Investigation, vol. 30, no. 1, pp. 15-34, 2010.

[20] P. V. Ruitenbeek, A. Vermeeren, and W. J. Riedel, "Histamine H1 receptor antagonist cetirizine impairs working memory processing speed, but not episodic memory," British Journal of Pharmacology, vol. 161, no. 2, pp. 456-466, 2010. 


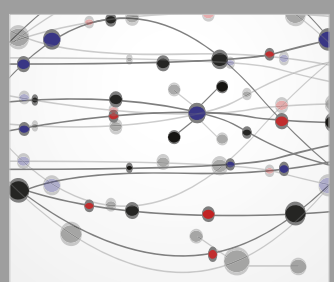

The Scientific World Journal
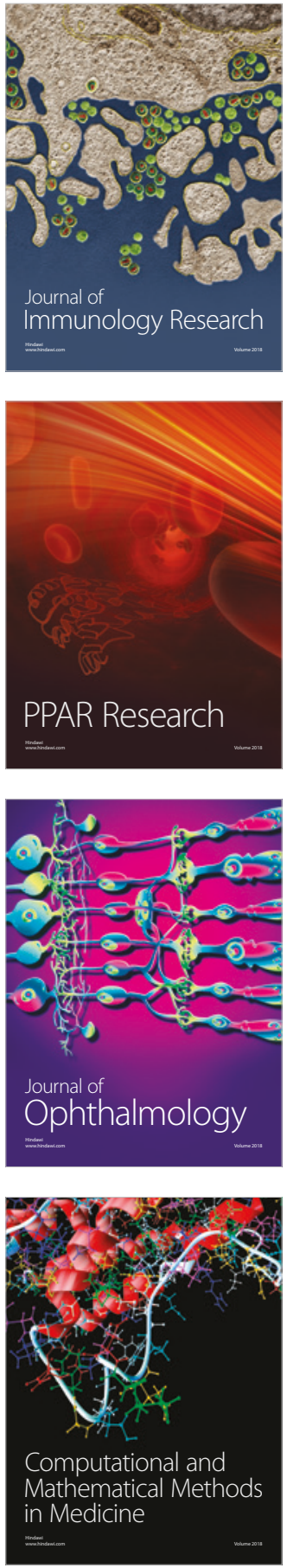

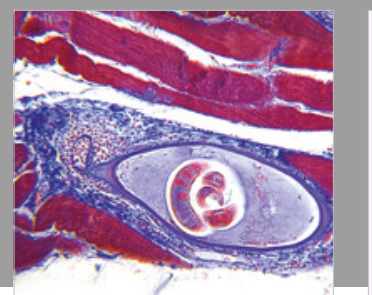

Gastroenterology Research and Practice

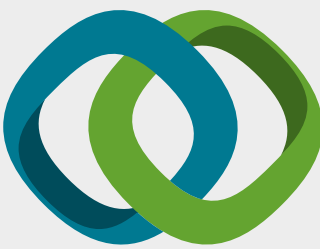

\section{Hindawi}

Submit your manuscripts at

www.hindawi.com
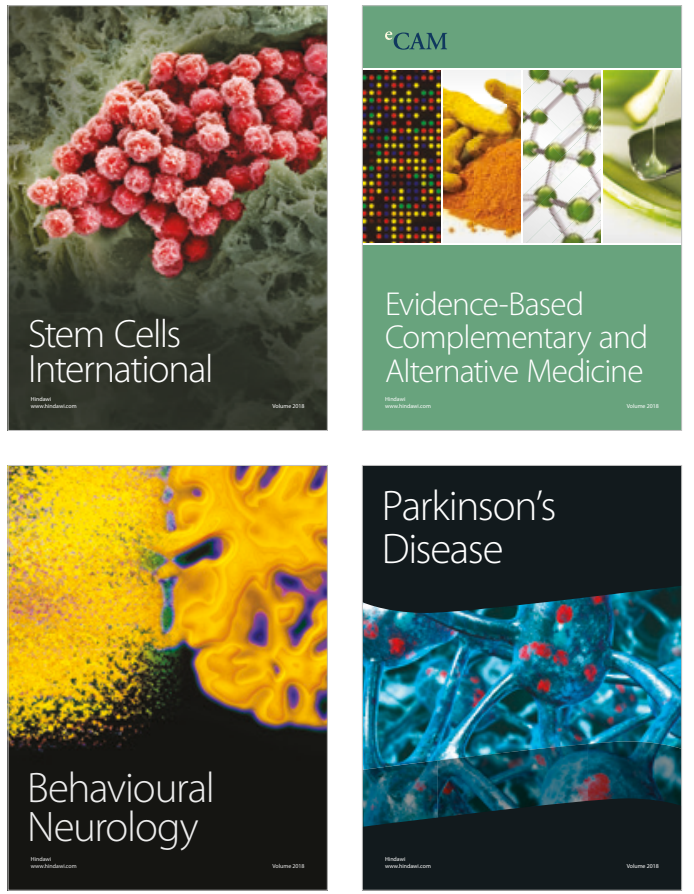

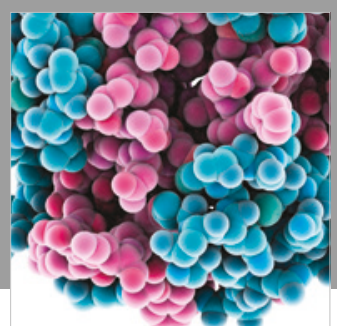

ournal of

Diabetes Research

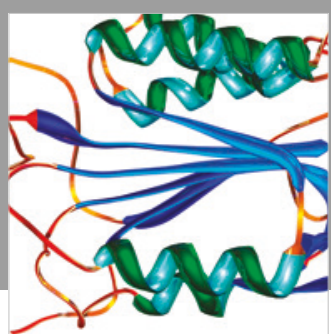

Disease Markers
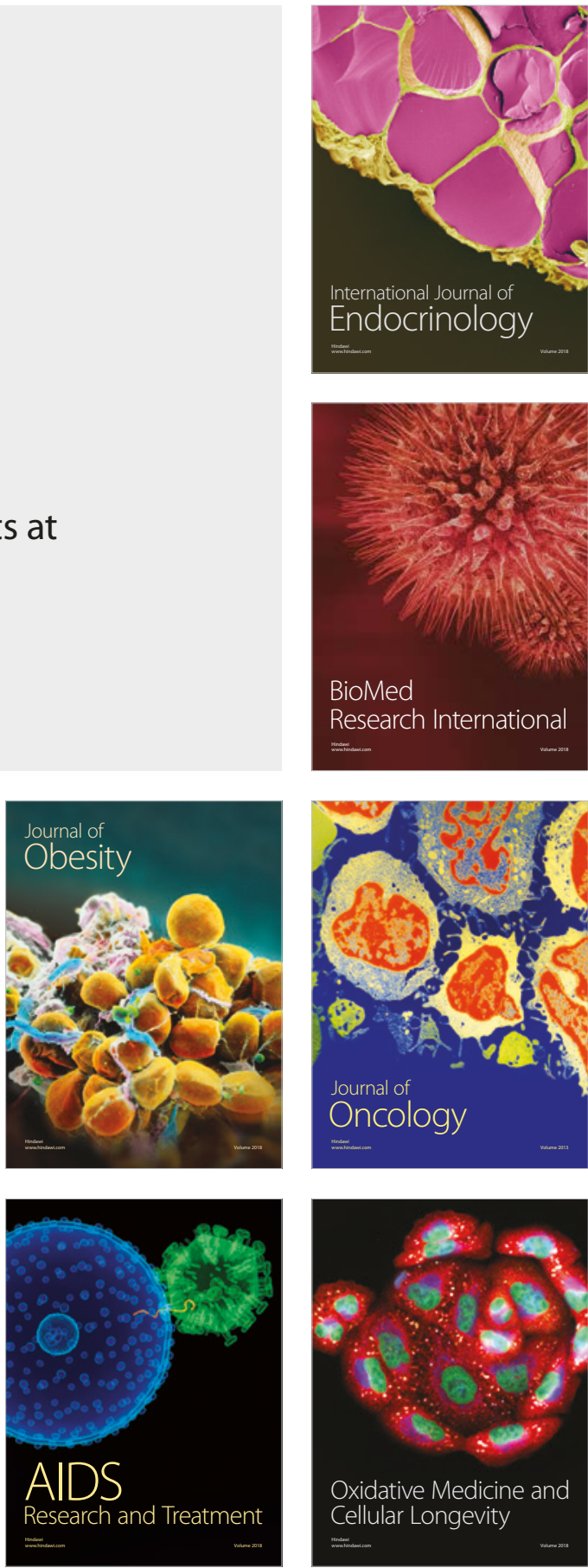